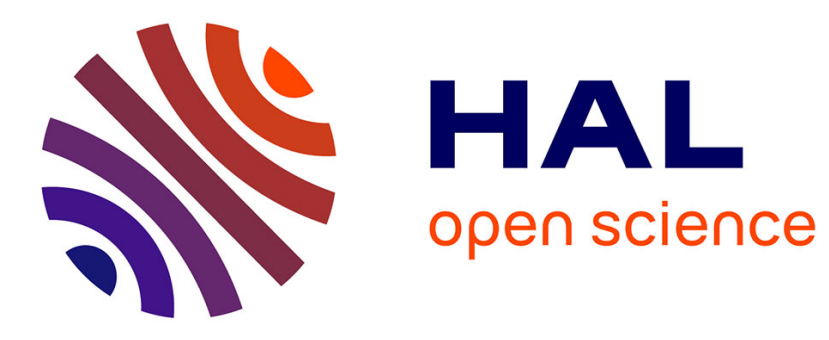

\title{
Hydroxyl radical production induced by plasma hydrogenated nanodiamonds under X-ray irradiation
}

Magdalena Kurzyp, Hugues Girard, Yannis Cheref, Emilie Brun, Cécile Sicard-Roselli, Samuel Saada, Jean-Charles Arnault

\section{- To cite this version:}

Magdalena Kurzyp, Hugues Girard, Yannis Cheref, Emilie Brun, Cécile Sicard-Roselli, et al.. Hydroxyl radical production induced by plasma hydrogenated nanodiamonds under X-ray irradiation. Chemical Communications, 2017, 53 (7), pp.1237 - 1240. 10.1039/c6cc08895c . hal-01879699

\section{HAL Id: hal-01879699 \\ https://hal.science/hal-01879699}

Submitted on 22 Jul 2019

HAL is a multi-disciplinary open access archive for the deposit and dissemination of scientific research documents, whether they are published or not. The documents may come from teaching and research institutions in France or abroad, or from public or private research centers.
L'archive ouverte pluridisciplinaire HAL, est destinée au dépôt et à la diffusion de documents scientifiques de niveau recherche, publiés ou non, émanant des établissements d'enseignement et de recherche français ou étrangers, des laboratoires publics ou privés. 


\title{
Hydroxyl Radicals Production Induced by Plasma Hydrogenated Nanodiamonds under X-ray Irradiation
}

\author{
Magdalena Kurzyp ${ }^{a}$, Hugues A. Girard ${ }^{a}$, Yannis Cheref ${ }^{b}$, Emilie Brun ${ }^{b}$, Cecile Sicard-Roselli ${ }^{b}$, Samuel Saada ${ }^{a}$ and Jean-Charles \\ Arnault $^{\mathrm{a}}$
}

a. CEA, LIST, Diamond Sensors Laboratory, F-91191 Gif-sur-Yvette, France

E-mail: jean-charles. arnault.cea.fr

b. University Paris-Sud, Laboratory of Physical Chemistry, CNRS UMR 8000, Orsay, F-91405, France

E-mail: cecile.sicard@u-psud.fr

For the first time, overproduction of hydroxyl radicals $\left(\mathrm{HO}^{\circ}\right)$ induced by plasma hydrogenated detonation nanodiamonds $(\mathrm{H}-$ NDs) under X-ray irradiation is reported. Using coumarin (COU) as a fluorescent probe, we reveal a significant increase of $40 \%$ of the $\mathrm{HO}^{\circ}$ production in presence of $\mathrm{H}-\mathrm{NDs}(6-100 \mu \mathrm{g} / \mathrm{ml})$ compared to water alone. This effect is related to the negative electron affinity of the hydrogenated nanodiamonds and illustrates the ability of H-NDs to produce reactive oxygen species probably via electron emission in water under $\mathrm{X}$-rays irradiation.

Detonation nanodiamonds (NDs) among all carbon nanomaterials possess outstanding chemical and physical properties to be used in many cross-disciplinary applications. Their small size ${ }^{1}$, hardness and low friction coefficient ${ }^{2}$, chemical resilience ${ }^{3}$, thermal conductivity ${ }^{4}$, colloidal stability ${ }^{5}$ and biocompatibility make NDs an appropriate material for nanocomposites 6 , sensors 7 , nanophotonics but also for bio- and electrochemical uses ${ }^{8,9}$. In addition to that, well-controlled mass production and tuned-surface chemistry make NDs widely available and versatile material.

The miscellaneous chemical surface obtained after production brought end-users to develop techniques to control NDs' surface e.g. homogenisation ${ }^{10}$. Mass treatments (air or vacuum annealing, wet chemistry and plasma activation) leading to oxidised ${ }^{11}$, hydrogenated ${ }^{3}$ and graphitised ${ }^{12}$ NDs have been mostly explored as well as NDs corresponding properties and behaviours ${ }^{10,13}$. Focusing only on the hydrogen-terminated surface, homogenisation of the NDs introduced via annealing or plasma treatment leads to a modification of their colloidal properties ${ }^{3,14}$ resulting in positive zeta potential (ZP) in aqueous solution ${ }^{5}$ providing colloidal stability $5,15,16$. At the same time, hydrogen surface homogenisation of the NDs drives to a modification of their electronic structure. Bolker et al. demonstrated that the particles exhibit a negative electron affinity (NEA) ${ }^{17}$ after a plasma hydrogenation similar to the one observed for bulk hydrogenated diamond materials. Kondo et al. showed that electrical conductivity of NDs appears when hydrogen is successfully incorporated by thermal annealing on the surface of nanodiamonds ${ }^{18}$. This property originates from the surface transfer doping occurring by adsorption of molecules onto the hydrogenated diamond surface. In parallel, Robert Hammers' team recently started to investigate the electron emission properties in water of hydrogenated diamond materials ${ }^{19,20,21}$. For instance, in the pioneering paper of Zhu et al. ${ }^{22}$, they reported that hydrogenated bulk diamond electrodes can be used as a solid-state source of solvated electrons in water upon UV excitation. These solvated electrons can then induce novel reactions such as $\mathrm{CO}_{2}$ reduction to $\mathrm{CO}^{19}$ or $\mathrm{N}_{2}$ to $\mathrm{NH}_{3}{ }^{23}$. Concerning nanodiamonds, such electron emission in water from hydrogenated particles has not been directly investigated so far but indirect hints arise from recent publication related to therapeutic applications of H-NDs. For instance, in 2015 Grall et al. showed that more cell death was induced in radioresistant cancer cells when combining H-NDs and gamma rays correlated to more reactive oxygen species measured after 1 and $24 \mathrm{~h}$ after irradiation ${ }^{24,25}$.

In this context of promising prospects around the hydrogenated diamond as a source of solvated electrons and to potential new applications such as radiotherapy enhancer for nanodiamonds, this paper aims to investigate the creation of hydroxyl radicals $\left(\mathrm{HO}^{\circ}\right)$ in water from $\mathrm{H}-\mathrm{NDs}$ under $\mathrm{X}$-Ray radiation using coumarin as a probe. $\mathrm{HO}^{\bullet}$ are among the major products of water radiolysis, however, they are characterised by a short lifetime (ns range) and low diffusion range often inducing indirect detection and quantification ${ }^{26}$. Since 1965, coumarin and its non-hydroxylated derivatives have been studied showing weak fluorescence properties but in the presence of $\mathrm{HO}^{\circ}, \mathrm{COU}$ immediately hydroxylates giving several hydroxycoumarins, among which only $7-\mathrm{OH} \mathrm{COU}$ possesses a high fluorescence yield ${ }^{27}$. This property has been widely used with other nanoparticles (NPs), like gold ${ }^{28,29}$, for detection and quantification of $\mathrm{HO}^{\circ}$ due to its simplicity, selectivity and high sensitivity.

The $5 \mathrm{~nm}$ detonation nanodiamonds powder provided by PlasmaChem Company (Germany) were modified via air annealing and microwave plasma hydrogenation leading to carboxylated NDs (Ox-NDs) and hydrogenated NDs (H-NDs), respectively. Ox-NDs were used as a reference material because electron emission is not expected due to its positive electron affinity (PEA) ${ }^{30}$. Both materials were dispersed in ultra-pure water by sonication and centrifugation [†ESI] in order to obtain two stable colloidal suspensions possessing similar colloidal properties. The obtained NDs water suspensions were characterised in terms of hydrodynamic radius, ZP and surface terminations via DLS measurements and FTIR spectroscopy. XPS and Raman characterizations of plasma hydrogenated nanodiamonds were previously reported ${ }^{5,31}$. 
The hydrodynamic diameter of NDs aggregates proved to be $33 \pm$ $15 \mathrm{~nm}$ and $38 \pm 14 \mathrm{~nm}$ with ZP of $-53 \pm 10 \mathrm{mV}$ and $+44 \pm 7 \mathrm{mV}$, for Ox-NDs and H-NDs, respectively [TESI S1 and S2]. FTIR spectra showed that $\mathrm{Ox}-\mathrm{ND}$ possesses a strong peak related to $\mathrm{C}=\mathrm{O}$ groups $\left(1730-1750 \mathrm{~cm}^{-1}\right)$ where the hydrogen treatments remove $\mathrm{C}=0$ groups enhancing vibrations in $2800-3000 \mathrm{~cm}^{-1}$ range associated with $\mathrm{C}-\mathrm{H}$ stretching [ $\mathrm{tESI} \mathrm{S3}$ ]. Results obtained are in agreement with previous papers dealing with properties of the oxidised and hydrogenated nanodiamonds ${ }^{11}$.
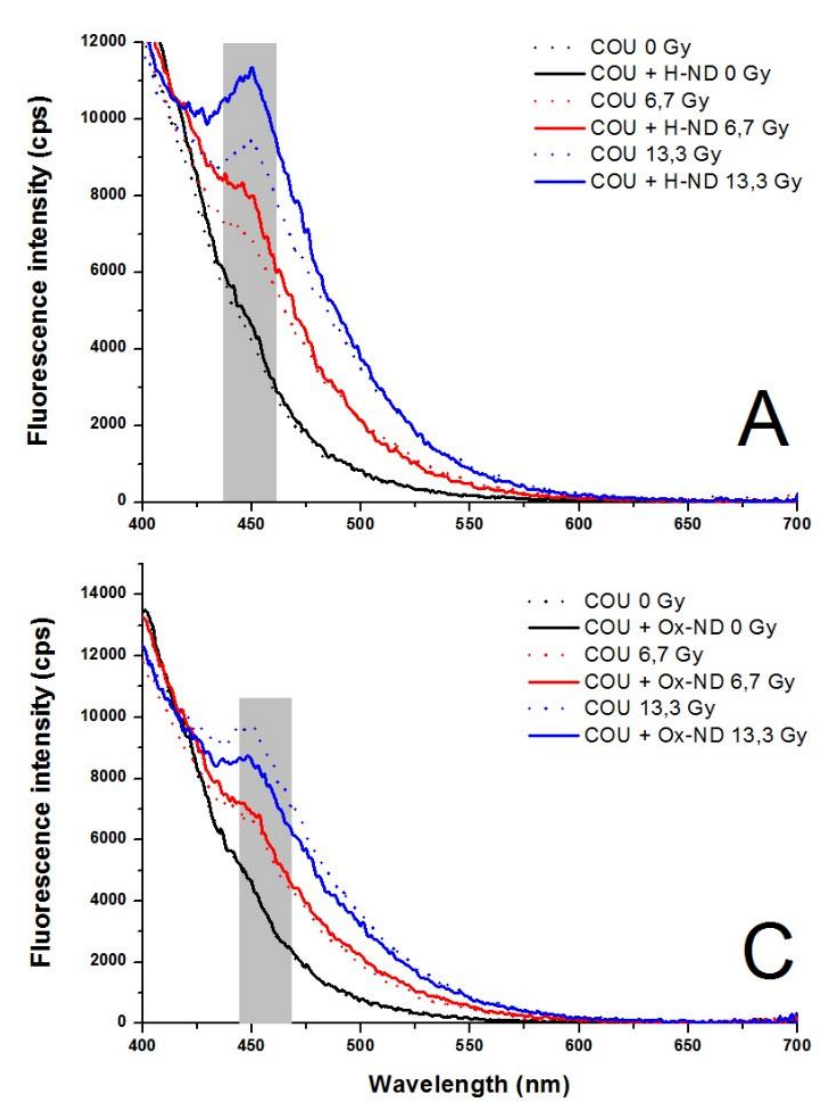

Fig.1. (A and C) Representative data of fluorescence spectra for coumarin (dash line) and coumarin with $30 \mu \mathrm{g} / \mathrm{ml}$ of H-NDs (A) and Ox-ND (C) (solid line). Data collected for both non-irradiated (black) and irradiated samples of two representative doses of $X$ ray irradiation (6.7 Gy - red and $13.3 \mathrm{~Gy}$ - blue). The highlighted grey area indicates part of the spectrum with the highest $7-0 \mathrm{OH}$ COU fluorescence intensity, the maximum was recorded at 452 $\mathrm{nm}$ and used for further $\mathrm{HO}^{\circ}$ quantification.

Graphs $B$ and $D$ depict the 7-hydroxycoumarin concentration given in $\mathrm{nM}$ according to the irradiated dose (Gy) for H-ND (B) and OxND (D) at 6, 10 and $30 \mu \mathrm{g} / \mathrm{mL}$. Slopes of linear fits represent the Gvalue of $7-\mathrm{OH} \mathrm{COU}$ in $\mathrm{nM} / \mathrm{J}$.

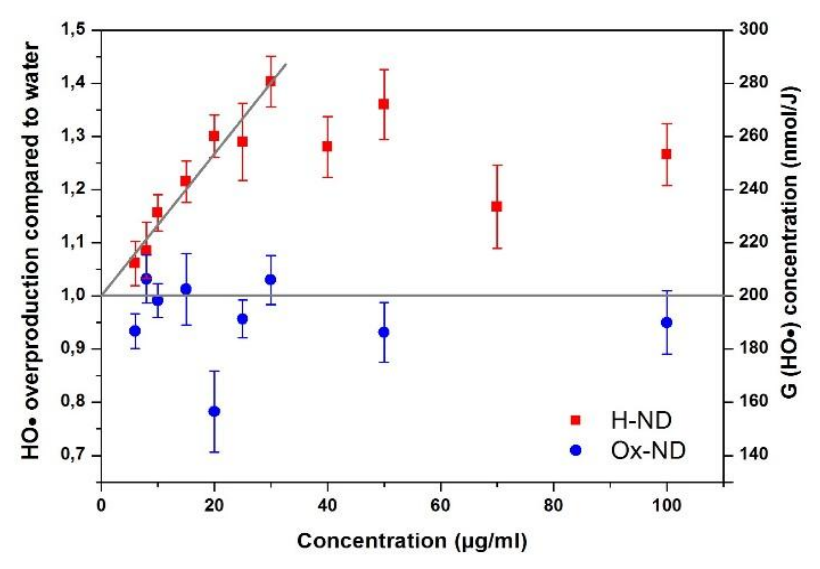

Ox-NDs and H-NDs (concentration up to $100 \mu \mathrm{g} / \mathrm{ml}$ ) were then mixed with $\mathrm{COU}(0.5 \mathrm{mM})$ and exposed to non-monochromatic $\mathrm{X}$ rays (Molybdenum cathode, $\mathrm{E}=17.5 \mathrm{keV}, 20 \mathrm{~Gy} / \mathrm{min}$ dose rate). NDs were irradiated with increasing doses up to $13.3 \mathrm{~Gy}$. To ensure that 7-OH COU fluorescence signal was not disturbed by the presence of NDs particles, $12 \mu \mathrm{L}$ of $\mathrm{NaCl}$ solution $(10 \%, \mathrm{w} / \mathrm{v})$ were added into post-irradiated samples to induce their self-aggregation and samples were centrifuged (18 000g, $5 \mathrm{~min}$ ). [tESI S4] Fluorescence of the supernatant was then read with an excitation
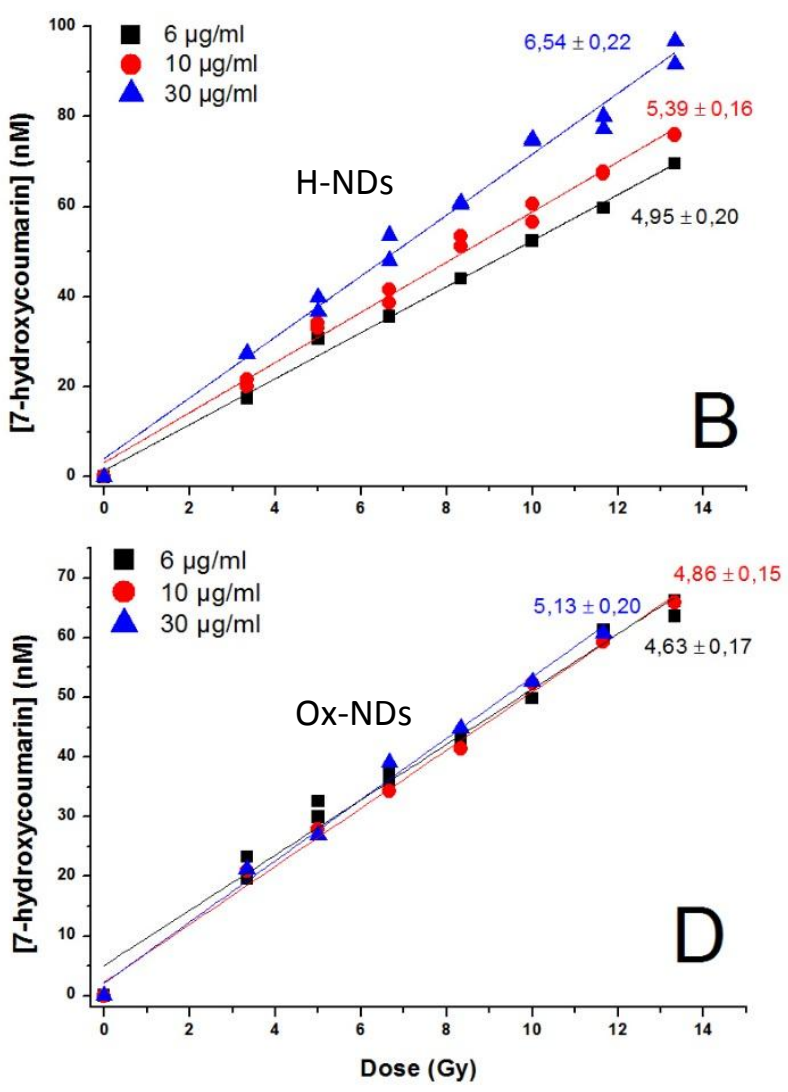

wavelength of $326 \mathrm{~nm}$ and emission spectra recorded between 380 and $700 \mathrm{~nm}$. To establish the link between the fluorescence intensity and the 7-OH COU, calibration samples of 7-OH $\mathrm{COU}$ diluted in $\mathrm{COU}(0.5 \mathrm{mM})$ were prepared [tESI S4]. Fig. $1 \mathrm{~A}$ and $1 \mathrm{C}$ report the fluorescence spectra recorded for samples before and after irradiation in the presence of $30 \mu \mathrm{g} / \mathrm{ml}$ of Ox-NDs or H-ND for two different doses. Before irradiation, almost no fluorescence signal is detected around $450 \mathrm{~nm}$. After irradiation of COU without NDs, a small peak can be seen at $452 \mathrm{~nm}$, which intensity depends on the dose. Furthermore, in presence of H-NDs (Fig. 1A), this fluorescence signal is exalted, whereas in presence of Ox-NDs it remains the same (Fig. 1C).

Fluorescence spectra of different NDs concentrations and X-ray doses were obtained and the peak intensity at $452 \mathrm{~nm}$ (corrected from the background) was translated into $7-\mathrm{OH} \mathrm{COU}$ concentrations through the calibration curve [tESI S4]. Figures $1 \mathrm{~B}$ and $1 \mathrm{D}$ show 7$\mathrm{OH} \mathrm{COU}$ concentrations obtained for different H-ND and Ox-NDs concentrations according to X-ray dose. Without NDs, the linear 
increase of 7-OH COU concentration according to the X-ray dose depicts the standard radiolysis phenomenon. In the presence of NDs, the linear increase is preserved, and the slopes tend to rise according to accumulative H-NDs concentration, while they stay relatively stable with some small fluctuations within an error bar in the presence of Ox-NDs. Both findings: the linearity of the curves and the slope evolution, observed only for H-NDs, reveal that an overproduction of 7-OH COU occurs only for hydrogen-terminated nanoparticles. This supports the hypothesis of non-photoactivity of Ox-NDs.

Fig.2. Hydroxyl radicals overproduction as a function of ND concentration for H-ND (red) and Ox-ND (blue). The left $Y$-axis shows normalization to water radiolysis. The right $Y$-axis shows the corresponding G-values in $\mathrm{nmol} / \mathrm{J}$ for $\mathrm{HO}^{\circ}$ radicals. The horizontal line represents production in water.

Slope values of all these experiments were then divided by the slope for coumarin only and plotted according to the NDs concentration. (Fig. 2) For each concentration, at least 2 independent experiments with 8 different doses each time were taken into account. Note that all these slopes represent the formation yield of 7-OH COU, also called G-value. At ND concentration $=0 \mu \mathrm{g} / \mathrm{ml}$, the value of 1 describes the yield of $7-\mathrm{OH}$ COU occurring during the water radiolysis, which corresponds to a known value of $\mathrm{HO}^{\circ}$ in the conditions used, and equal to $200 \mathrm{nmol} / \mathrm{J}$ (horizontal grey line). Thanks to this conversion, a second scale reporting the direct $\mathrm{HO}^{\circ}$ production yield can be added to Figure 2 . This first part of the curve definitely supports the hypothesis about the photoemission from the H-NDs surface leading to the overproduction of $\mathrm{HO}^{\circ}$, with a concentration dependence of the phenomenon (diagonal grey line). The maximum value obtained is $40 \%$ higher compared to the water radiolysis alone at a concentration of $30 \mu \mathrm{g} / \mathrm{mL}$ of H-NDs. Ox-NDs appear here as a negative control, the experiments show no $\mathrm{HO}^{\circ}$ overproduction and hence a lack of electron emission from the carboxylated surface due to their PEA.

Secondly, above $30 \mu \mathrm{g} / \mathrm{ml}$ of $\mathrm{H}-\mathrm{NDs}$, the $\mathrm{G}\left(\mathrm{HO}^{\circ}\right)$ values seem to saturate. The reason of that is not elucidated so far and maybe linked to recombination of radicals ${ }^{28}$. Another related option can rely on the oxidation of the $\mathrm{H}-\mathrm{NDs}$ under the production of highly reactive $\mathrm{HO}^{\circ}$. Abstraction of hydrogen atoms by hydroxyl radicals has already been proposed in the literature, leaving dangling bonds which can react with water molecules forming some oxidized terminations ${ }^{23}$. The small decrease of the fluorescence signal can also be linked to a quenching effect, often observed for fluorescence measurements in presence of NPs. However, as shown in figure ESI S4, by the addition of salt and centrifugation of the post-irradiated samples, $95 \%$ of the fluorescence can be recovered. Consequently, the decline in the $\mathrm{G}\left(\mathrm{HO}^{\circ}\right)$ signal seems to be directly related to the specific $\mathrm{H}-\mathrm{ND}$ surface properties.

The most important finding to emerge from this study is that NEA of $\mathrm{H}$-NDs gives rise to/ is correlated to measurable overproduction of ROS in the aqueous environment, most probably resulting from emission of electrons under X-Ray. In this paper, we investigated only hydroxyl radicals which are the major deleterious species in a biological environment supplying the concept of electron emission from the hydrogen-terminated NDs. Same results were obtained with different commercial sources of detonation NDs (e.g. Adamas,
USA and NanoCarbon Research Institute, Japan), exhibiting different level and nature of impurities, proving that the electron emission is source independent and depends only on $-\mathrm{H}$ terminations of the surface of NPs.

To conclude, for the first time, the overproduction of $\mathrm{HO}^{\circ}$ in the presence of $\mathrm{H}$-NDs under biologically relevant doses of low keV Xray irradiation is reported. A significant increase of $+40 \%$ of the 7 $\mathrm{OH} \mathrm{COU} \mathrm{production} \mathrm{in} \mathrm{the} \mathrm{presence} \mathrm{of} 30 \mu \mathrm{g} / \mathrm{ml} \mathrm{H-ND}$ is observed. These results support that electron production is enhanced on $\mathrm{H}$ terminated NDs treated by microwave hydrogen plasma. This effect is also coherent with NEA property of $5 \mathrm{~nm}$ NDs. In addition, this effect is not observed for Ox-NDs where electron emission is less favourable due to PEA ${ }^{30}$. These results obtained for ND concentrations and X-ray doses compatible with biological studies, confirm the promising utility of $\mathrm{H}-\mathrm{NDs}$ as radiosensitizers.

\section{Acknowledgements}

The authors would like to acknowledge Stéphanie Droniou (University Paris-Sud, Laboratory of Physical Chemistry, CNRS UMR 800, Orsay, F-91405, France) for assistance during X-ray irradiation of the samples. This work is founded by the CFR program by CEA and the CEA internal program NEXT as a part of the NanoScience and Technology pour la Santé CEA-Transverse initiative. 


\section{Notes and references}

1

O. A. Williams, J. Hees, C. Dieker, W. Jäger, L. Kirste and C. E. Nebel, ACS Nano, 2010, 4, 48244830.

M. Ivanov, Z. Mahbooba, D. Ivanov, S. Smirnov, S. Pavlyshko, E. Osawa, D. Brenner and O. Shenderova, 2014, 5, 160-166.

H. A. Girard, T. Petit, S. Perruchas, T. Gacoin, C. Gesset, J. C. Arnault and P. Bergonzo, Phys. Chem. Chem. Phys., 2011, 13, 11517-23.

E. Roumeli, E. Pavlidou, A. Avgeropoulos, G. Vourlias, D. N. Bikiaris and K. Chrissafis, J. Phys. Chem. B, 2014, 118, 11341-11352.

T. Petit, H. A. Girard, A. Trouvé, I. BatonneauGener, P. Bergonzo and J.-C. Arnault, Nanoscale, 2013, 5, 8958-62.

V. N. Mochalin and Y. Gogotsi, Diam. Relat. Mater., 2015.

R. Schirhagl, K. Chang, M. Loretz and C. L. Degen, Annu. Rev. Phys. Chem, 2014, 65, 83105.

L.-W. Tsai, Y.-C. Lin, E. Perevedentseva, A. Lugovtsov, A. Priezzhev and C.-L. Cheng, 2016.

V. N. Mochalin, O. Shenderova, D. Ho and Y. Gogotsi, Nat. Nanotechnol., 2012, 7, 11-23.

A. Krueger and D. Lang, Adv. Funct. Mater., 2012, 22, 890-906.

J.-C. Arnault, T. Petit, H. Girard, A. Chavanne, C. Gesset, M. Sennour and M. Chaigneau, Phys. Chem. Chem. Phys., 2011, 13, 11481-7.

J.-C. Arnault, T. Petit, H. A. Girard, C. Gesset, M. Combis-Schlumberger, M. Sennour, A. Koscheev, A. A. Khomich, I. Vlasov and O. Shenderova, Phys. status solidi, 2014, 211, 2739-2743.

J.-C. Arnault, Nianjun Yang Editor, Novel Aspects of Diamond, Springer, 2014, Chapter 5, 85-122.

J.-C. Arnault and H. A. Girard, Current Opinion in Solid State and Materials Science, 2016 doi:10.1016/j.cossms.2016.06.007.
Aziz, Nanoscale, 2015, 7, 2987-2991.

S. Stehlik, T. Glatzel, V. Pichot, R. Pawlak, E. Meyer, D. Spitzer and B. Rezek, Diam. Relat. Mater., 2015.

A. Bolker, C. Saguy and R. Kalish, Nanotechnology, 2014, 25, 385702.

T. Kondo, I. Neitzel, V. N. Mochalin, J. Urai, M. Yuasa and Y. Gogotsi, J. Appl. Phys., 2013, 113.

L. Zhang, D. Zhu, G. M. Nathanson and R. J. Hamers, Angew. Chemie - Int. Ed., 2014, 97469750.

\section{R. J. Hamers, J. a Bandy, D. Zhu and L. Zhang,} Faraday Discuss., 2014, 172, 1-15.

R. J. Hamers and J. Bandy, Phys. status solidi, 2016, 213, 2069-2074.

D. Zhu, L. Zhang, R. E. Ruther and R. J. Hamers, Nat. Mater., 2013, 12, 836-841.

D. Zhu, J. A. Bandy, S. Li and R. J. Hamers, Surf. Sci., 2016, 650, 295-301.

T. Petit, H. A. Girard, M. Combis-Schlumberger, R. Grall, J. Delic, S. Morel-Altmeyer, P. Bergonzo, S. Chevillard and J. C. Arnault, Proc. IEEE Conf. Nanotechnol., 2013, 174-178.

R. Grall, H. A. Girard, L. Saad, T. Petit, C. Gesset, M. Combis-Schlumberger, V. Paget, J. Delic, J.-C. Arnault and S. Chevillard, Biomaterials, 2015, 61, 290-298.

G. L. Newton and J. R. Milligan, Radiat. Phys. Chem., 2006, 75, 473-478.

G. Louit, S. Foley, J. Cabillic, H. Coffigny, F. Taran, A. Valleix, J. P. Renault and S. Pin, Radiat. Phys. Chem., 2005, 72, 119-124.

C. Sicard-Roselli, E. Brun, M. Gilles, G. Baldacchino, C. Kelsey, H. McQuaid, C. Polin, N. Wardlow and F. Currell, Small, 2014, 10, 333846.

M. Gilles, E. Brun and C. Sicard-Roselli, Colloids Surfaces B Biointerfaces, 2014, 123, 770-777.

T. Petit, J.-C. Arnault, H. a Girard, M. Sennour, T.-Y. Kang, C.-L. Cheng and P. Bergonzo, Nanoscale, 2012, 4, 6792-9. 
31 M. Mermoux, A. Crisci, T. Petit, H. A. Girard and

J.-C. Arnault, J. Phys. Chem. C, 2014, 118,

23415-23425. 